\title{
Contents to Volume 125 (2001)
}

Volume 125 No. 1, October 2000

Laurent Moret-Bailly: Problèmes de Skolem sur les champs algébriques

Giorgio Ottaviani and Raffaella Paoletti: Syzygies of Veronese Embeddings

Nikolaos Diamantis: Hecke Operators and Derivatives of $L$-Functions

Yoshinori Namikawa: Global Smoothing of Calabi-Yau Threefolds II

Eric M. Friedlander and Mark E. Walker: Function Spaces and Continuous Algebraic Pairings for Varieties

Kiyoshi Takeuchi: Microlocal Vanishing Cycles and Ramified Cauchy Problems in the Nilsson Class

$111-127$

\section{Volume 125 No. 2, January (II) 2001}

Jesús A. Álvarez López and Yuri A. Kordyukov: Long Time Behavior of Leafwise Heat Flow for Riemannian Foliations

Bernhard Krötz: Formal Dimension for Semisimple Symmetric Spaces

Marc Chardin, David Eisenbud and Bernd Ulrich: Hilbert Functions, Residual Intersections, and Residually $S_{2}$ Ideals

T. N. Venkataramana: Cohomology of Compact Locally Symmetric Spaces 


\section{Volume 125 No. 3, February 2001}

Jun-Muk Hwang: On the Vanishing Orders of Vector Fields on Fano Varieties of Picard Number 1*

Peter Vermeire: Some Results on Secant Varieties Leading to a Geometric Flip Construction

Philippe Gille: Cohomologie galoisienne des groupes quasi-déployés sur des corps de dimension cohomologique $\leqslant 2$

Alexei Bondal and Dmitri Orlov: Reconstruction of a Variety from the Derived Category and Groups of Autoequivalences ${ }^{\star}$

Jianxun Hu: Gromov-Witten Invariants of Blow-ups Along Surfaces

David B. Massey: The Sebastiani-Thom Isomorphism in the Derived Category

Erratum: Jan Denef and Akihiro Gyoja: Character Sums Associated to Prehomogeneous Vector Spaces, Composito Math. 113 (3) (1998), 273-346

Author Index to Volume 125 (2001) 